\title{
FUZZY LINEAR FRACTIONAL SET COVERING PROBLEM WITH IMPRECISE COSTS
}

\author{
Rashmi Gupta $^{1}$ and Ratnesh Rajan Saxena ${ }^{2}$
}

\begin{abstract}
Set covering problems are in great use these days, these problems are applied in many disciplines such as crew scheduling problems, location problems, testing of VLSI circuits, artificial intelligence etc. In this paper $\alpha$-acceptable optimal solution is given for the fuzzy linear fractional set covering problem where fuzziness involved in the objective function. At first the fuzzy linear fractional problem is being converted in to crisp parametric linear fractional set covering problem then a linearization technique is used to obtain an optimal solution to this parametric problem. This optimal solution will also be the fuzzy optimal solution for the original problem. An example is also provided to illustrate the algorithm.
\end{abstract}

Keywords. Fuzzy fractional set covering problem, $\alpha$-optimal solution, fuzzy solution.

Mathematics Subject Classification. 90C70, 90C10.

\section{INTRODUCTION}

Mathematical programming problems finds an extreme use in a large number of domains. An important class of mathematical programming is Fractional Set Covering Problems which tackles the situations where a ratio of functions is to be minimized. Fractional Programming has attracted the attention of many

Received September 2, 2013. Accepted February 24, 2014.

1 Department of Mathematics, University of Delhi, 110007 Delhi, India. smsrashmigupta@gmail.com

2 Department of Mathematics, Deen Dayal Upadhyaya College (University of Delhi), Karampura, 110015 New Delhi, India. ratnesh65@gmail.com 
researchers in the past but not much attention is being given to fuzzy fractional set covering problems though they have many applications in real life. Scheduling is a major application of the set covering problems. Other application areas are for example resource allocation, pattern recogonization and machine learning. Recently fuzzy set covering problem have been studied by Saxena and Gupta $[11,12]$, Stanojević and Stanojević [14], Huang et al. [2], Sahraeian and Sdeq Kazemi [9], Shavandi and Mahlooji [13], Zimmermann [17], Li and Kwan [5,6]. They discussed various approaches for solving set covering problems. In this paper our objective is to consider a fuzzy linear fractional set covering problem with fuzzy cost coefficient and develop an algorithm which provides a range in the solution for the decision maker i.e. a fuzzy solution.

Often, a decision maker is supposed to be able to exactly fit all numerical values taking part in the problem, but frequently he does not know those exact values, rather he knows some approximations of them and therefore he may feel more comfortable in specifying vague (fuzzy) values rather than point values. This paper considered the case in which a decision maker has a set covering problem in which the coefficients defining the objective function are given by fuzzy numbers whereas the constraints set is a conventional one. Thus the existence of the membership function is assumed. These membership functions will capture the knowledge that the decision maker has about each cost taking part in to account.

The paper has the following structure. In Section 2, Fuzzy Linear Fractional Set Covering Problems and some definitions are presented. In Section 3, algorithm is presented to solve the given fuzzy linear fractional set covering problem. In Section 4, numerical example is given in support of the proposed algorithm. Section 5, concludes the paper.

\section{THEORETICAL DEVELOPMENT}

\section{LineAR SET COVERING PROBLEMS (CP)}

Set covering problem is a classical problem in computer science and complexity theory and is one of the most important discrete optimization problem because it serves as a model for real world problems. Real world problem that can be modeled as set covering problem include facility location problem and airline crew scheduling problem, etc.

Analytically the problem is, consider a set $I=\{1,2, \ldots, m\}$ and a set $P=$ $\left\{P_{1}, P_{2}, \ldots, P_{n}\right\}$, where $P_{j} \subseteq I$ for each $j \in J=\{1,2, \ldots, n\}$. A subset $J^{*}$ of $J$ is said to be a cover of $I$ if $\bigcup_{j \in J^{*}} P_{j}=I$. Let a cost $c_{j}>0$ be associated with every $j \in J$. Then the total cost of the cover $J^{*}$ is equal to $\sum_{j \in J^{*}} c_{j}$.

The linear set covering problem $(\mathrm{CP})$ is to find a cover of minimum cost subject to the condition that at least one of the utility is satisfied. Mathematically, the 
problem is

$$
\begin{aligned}
\min f(x) & =\sum_{j=1}^{n} c_{j} x_{j} \\
\text { subject to } \sum_{j=1}^{n} a_{i j} x_{j} & \geq 1, \quad i \in I \\
x_{j} & =0 \text { or } 1, \quad j \in J
\end{aligned}
$$

where $x_{j}=\left\{\begin{array}{ll}1 & \text { if } j \text { is in the cover } \\ 0 & \text { otherwise }\end{array}\right.$ and $a_{i j}= \begin{cases}1 & \text { if } i \in P_{j} \\ 0 & \text { otherwise. }\end{cases}$

In matrix form, $(\mathrm{CP})$ can be written as

$$
\begin{array}{r}
\min f(x)=c x \\
\text { subject to } A x \geq b,
\end{array}
$$

where $x^{T}=\left(x_{1}, x_{2}, \ldots, x_{n}\right)$ with $x_{j}=0$ or $1, j=1,2, \ldots, n$. Here $c=$ $\left(c_{1}, c_{2}, \ldots, c_{n}\right) \in \mathbb{R}^{n}$ is a row vector. $A$ is an $m \times n$ matrix of zeros and ones and $b^{T}=(1,1, \ldots, 1)$ is a row vector of ones.

The mathematical format of Linear Fractional Set-Covering Problem is

$$
\begin{gathered}
\min f(x)=\frac{\sum_{j=1}^{n} c_{j} x_{j}+p}{\sum_{j=1}^{n} d_{j} x_{j}+q} \\
\text { subject to } \sum_{j=1}^{n} a_{i j} x_{j} \geq 1, \quad i \in I \\
x_{j}=0 \text { or } 1, \quad j \in J,
\end{gathered}
$$

where $x_{j}=\left\{\begin{array}{ll}1 & \text { if } j \text { is in the cover } \\ 0 & \text { otherwise }\end{array}\right.$ and $a_{i j}= \begin{cases}1 & \text { if } i \in P_{j} \\ 0 & \text { otherwise. }\end{cases}$

It is assumed that $c_{j}$ 's and $d_{j}$ 's are non-negative numbers, $p$ and $q$ are constants such that $\sum_{j=1}^{n} d_{j} x_{j}+q>0$.

In matrix form, (LFP) can be written as

$$
\begin{aligned}
& \text { Minimize } f(x)=\frac{c x+p}{d x+q} \\
& \text { subject to } A x \geq b
\end{aligned}
$$

where $x^{T}=\left(x_{1}, x_{2}, \ldots, x_{n}\right)$ with $x_{j}=0$ or $1, j=1,2, \ldots, n$. Here $c=$ $\left(c_{1}, c_{2}, \ldots, c_{n}\right) \in \mathbb{R}^{n}$ and $d=\left(d_{1}, d_{2}, \ldots, d_{n}\right) \in \mathbb{R}^{n}$ are row vectors, $A$ is an $m \times n$ matrix of zeros and ones and $b^{T}=(1,1, \ldots, 1)$ is a row vector of ones, $q$ is a scalar such that $d x+q>0$. 


\section{FUZZY LINEAR FRACTIONAL SET COVERING PROBLEM (FLP)}

If the coefficient and the constants in the objective function of problem (LFP) becomes fuzzy in nature then problem (LFP) translates to fuzzy linear fractional set covering problem (FLP) i.e.

(FLP)

$$
\begin{aligned}
& \operatorname{Min} f(x)=\frac{\sum_{j=1}^{n} \widetilde{c}_{j} x_{j}+\widetilde{p}}{\sum_{j=1}^{n} \widetilde{d}_{j} x_{j}+\widetilde{q}} \\
& \text { subject to } \sum_{j=1}^{n} a_{i j} x_{j} \geq 1, \quad i \in I \\
& x_{j}=0 \text { or } 1, \quad j \in J,
\end{aligned}
$$

where $x_{j}=\left\{\begin{array}{ll}1 & \text { if } j \text { is in the cover } \\ 0 & \text { otherwise }\end{array}\right.$ and $a_{i j}= \begin{cases}1 & \text { if } i \in P_{j} \\ 0 & \text { otherwise. }\end{cases}$

It is assumed that $\widetilde{c}_{j}$ 's and $\widetilde{d}_{j}$ 's are non-negative and $\widetilde{q}$ is a scalar such that $\sum_{j=1}^{n} \widetilde{d}_{j} x_{j}+\widetilde{q}>0$.

In matrix form, (FLP) can be written as

$$
\begin{aligned}
& \text { Minimize } f(x)=\frac{\widetilde{c} x+\widetilde{p}}{\widetilde{d} x+\widetilde{q}} \\
& \text { subject to } A x \geq b,
\end{aligned}
$$

where $x^{T}=\left(x_{1}, x_{2}, \ldots, x_{n}\right)$ with $x_{j}=0$ or $1, j=1,2, \ldots, n$. Here $\widetilde{c}=$ $\left(\widetilde{c}_{1}, \widetilde{c}_{2}, \ldots, \widetilde{c}_{n}\right), \widetilde{d}=\left(\widetilde{d}_{1}, \widetilde{d}_{2}, \ldots, \widetilde{d}_{n}\right), \widetilde{p}=\left(\widetilde{p}_{1}, \widetilde{p}_{2}, \ldots, \widetilde{p}_{n}\right)$ and $\widetilde{q}=\left(\widetilde{q}_{1}, \widetilde{q}_{2}, \ldots, \widetilde{q}_{n}\right)$ are fuzzy row vectors, $A$ is an $m \times n$ matrix of zeros and ones and $b^{T}=(1,1, \ldots, 1)$ is a row vector of ones.

Following definitions are used in the development of the theory:

2.1 Cover Solution: a solution $X$ which satisfies (2.1) and (2.2) is said to be a cover solution.

2.2 Redundant Cover: given a cover $\mathcal{J}$ of $I$, a column $j^{*} \in \mathcal{J}$ is said to be redundant if $\mathcal{J}-j^{*}$ is also a cover. If a cover contains one or more redundant columns, it is called a redundant cover.

Note that a column $j^{*}$ is redundant with respect to the cover $\mathcal{J}$ iff $\sum_{j \in \mathcal{J}} a_{i j} \geq$ 2 for all $i \in P_{j^{*}}$.

2.3 Prime Cover: a cover $J^{*}$ is said to be a prime cover, if none of the columns corresponding to $j \in J^{*}$ is redundant. A solution corresponding to the prime cover is called a prime cover solution.

Following theorems forms the basis for the algorithm to enumerate (FLP).

Theorem 2.1 ([10]). If $J^{*}=\left\{j: x_{j}=1\right\}$ is any prime cover of (LFP) then $x=\left\{x_{j}\right\}$ is an extreme point of the convex set formed by feasible region. 
Theorem 2.2. If the objective function in (LFP) has finite value then, there exists a prime cover solution where this value is attained.

Proof. Let a finite optimal solution of (LFP) exists at $x_{0} \in S$ then the optimal value is

$$
f\left(x_{0}\right)=\frac{c x_{0}+p}{d x_{0}+q}
$$

Let $J_{0}$ be the cover corresponding to the solution $x_{0}$. If $J_{0}$ is the prime cover, then it is done, otherwise a prime cover can be derived from $J_{0}$ by dropping the redundant columns. Let $J_{1}$ be the prime cover obtained from $J_{0}$ and $x_{1}$ be the corresponding solution of (LFP) such that

$$
f\left(x_{1}\right)=\frac{c x_{1}+p}{d x_{1}+q}
$$

Since $c, d \geq 0, p$ and $q$ are positive numbers and $J_{1} \subseteq J_{0}$, therefore,

$$
\frac{c x_{1}+p}{d x_{1}+q} \leq \frac{c x_{0}+p}{d x_{0}+q}
$$

or

$$
f\left(x_{1}\right) \leq f\left(x_{0}\right)
$$

As $f\left(x_{0}\right)$ is the optimal value of $f(x)$, therefore, $f\left(x_{1}\right) \geq f\left(x_{0}\right)$. Hence $f\left(x_{1}\right)=$ $f\left(x_{0}\right)$. Which proves that there exist a prime cover solution yielding the optimal value of the objective function of (LFP).

Theorem 2.3 ([10]). Let $f(x)$ be a pseudo convex function defined on feasible set $S$ and $x^{*} \in S$ then $x^{*}$ is an optimal solution for the program

$$
\underset{x \in S}{\operatorname{Minimize}} f(x)
$$

if and only if, $x^{*}$ is an optimal solution for the program

$$
\underset{x \in S}{\operatorname{Minimize}} \nabla f\left(x^{*}\right)^{T} x
$$

where $S$ is the feasible region.

\section{Development of the Algorithm}

Let $F(\mathbb{R})$ denotes the set of all fuzzy numbers and let $\widetilde{r} \in F(\mathbb{R})$ with membership function $\mu_{\widetilde{r}}$ which assigns a real number $\mu_{\widetilde{r}}(x) \in[0,1]$ to each element, where the value $\mu_{\widetilde{r}}(x)$ at $x$ represents the grade of membership of $x$ in $\widetilde{r}$. Then $\widetilde{r}$ satisfies:

1. $\left\{x: x \in \mathbb{R}, \mu_{\widetilde{r}}(x)=1\right\} \neq \phi$ i.e., $\widetilde{r}$ is normal.

2. $\mu_{\widetilde{r}}$ is an upper semi-continuous quasi-concave function on $\mathbb{R}$.

3. Closure of the support set of $\mu_{\widetilde{r}}$, i.e. $\operatorname{cl}\left\{x \in \mathbb{R}: \mu_{\widetilde{r}}(x)>0\right\}$ is bounded. 
The $\alpha$-cut of the fuzzy number $\widetilde{r}$ is given by

$$
\widetilde{r}_{\alpha}=\left\{\begin{array}{ll}
\left\{x \mid x \in \mathbb{R}, \mu_{\widetilde{r}}(x) \geq \alpha\right\} & \text { if } \alpha \in(0,1] \\
\operatorname{cl}\left\{x \mid x \in \mathbb{R}, \mu_{\widetilde{r}}(x)>0\right\} & \text { if } \alpha=0
\end{array} .\right.
$$

It is clear from above that, for every $\alpha \in[0,1]$, the $\alpha$-cut set of $\widetilde{r}$ is a finite closed interval $\left[r_{\alpha}^{L}, r_{\alpha}^{R}\right]$ on $\mathbb{R}$. Let us call $\left[r_{\alpha}^{L}, r_{\alpha}^{R}\right]$ an $\alpha$-interval of confidence of the fuzzy number $\widetilde{r}$ which has a specific well-known meaning in fuzzy arithmetic.

Since the set $F(\mathbb{R})$ is not linearly ordered, in order to rank two fuzzy numbers in $F(\mathbb{R})$ we need to define a suitable ordering in $F(\mathbb{R})$. Dubois and Prade [1] proposed the possibility and the necessity indices to rank the fuzzy numbers. In this paper we make use of following possibility and necessity indices described by Dubois and Prade [1], and $\mathrm{Wu}[15]$.

We take the ordering between two fuzzy numbers, $\widetilde{r}, \widetilde{s} \in F(\mathbb{R})$, as follows: $\widetilde{r} \succeq_{\alpha} \widetilde{s}$, iff $\operatorname{Poss}(\widetilde{r} \succ \widetilde{s}) \geq \alpha$ and $\operatorname{Ness}(\widetilde{r} \succeq \widetilde{s}) \geq \alpha$.

The proof of the following proposition can be found in $\mathrm{Wu}$ [15].

Proposition 3.1. $\widetilde{r} \succeq_{\alpha} \widetilde{s}$, iff $r_{\alpha}^{L} \geq s_{\alpha}^{L}$ and $r_{\alpha}^{R} \geq s_{\alpha}^{R} \forall \alpha \in[0.5,1]$.

In what follows, we will write $\widetilde{s} \preceq_{\alpha} \widetilde{r}$, iff $\widetilde{r} \succeq_{\alpha} \widetilde{s}$. Also by $\widetilde{r} \prec_{\alpha} \widetilde{s}$, we will mean that $r_{\alpha}^{L}<s_{\alpha}^{L}$ and $r_{\alpha}^{R}<s_{\alpha}^{R}, \forall \alpha \in[0.5,1]$, and we will say, $\widetilde{r} \succ_{\alpha} \widetilde{s}$, iff $\widetilde{s} \prec_{\alpha} \widetilde{r}$.

A fuzzy number $\widetilde{r}$ will be called non-negative fuzzy number, denoted by $\widetilde{r} \succeq 0$, if for every $\alpha \in[0.5,1]$, we have $\left[r_{\alpha}^{L}, r_{\alpha}^{R}\right] \subset \mathbb{R}_{+}$, the non-negative real orthant. Also a fuzzy number $\widetilde{r}$ will be termed as positive fuzzy number, denoted by $\widetilde{r} \succ 0$, if for every $\alpha \in[0.5,1]$, we have, $\left[r_{\alpha}^{L}, r_{\alpha}^{R}\right] \subset \mathbb{R}_{+} \backslash\{0\}:=\mathbb{R}_{++}$.

Denote the sets of non-negative and positive fuzzy numbers, respectively by $F\left(\mathbb{R}_{+}\right)$and $F\left(\mathbb{R}_{++}\right)$.

Applying the interval arithmetic, some main operations for fuzzy numbers $\widetilde{r}, \widetilde{s} \in$ $F(\mathbb{R})$ are described as below (Kaufmann and Gupta [3], Zimmermann [17]).

1. Addition: $(\widetilde{r}+\widetilde{s})_{\alpha}=\left[r_{\alpha}^{L}+s_{\alpha}^{L}, r_{\alpha}^{R}+s_{\alpha}^{R}\right]$

2. Scalar multiplication: $(k \cdot \widetilde{r})_{\alpha}=\left[k r_{\alpha}^{L}, k r_{\alpha}^{R}\right]$ if $k>0$ and $(k \cdot \widetilde{r})_{\alpha}=\left[k r_{\alpha}^{R}, k r_{\alpha}^{L}\right]$ if $k<0$.

3. Multiplication:

$$
(\widetilde{r} \cdot \widetilde{s})_{\alpha}=\left[\min \left(r_{\alpha}^{L} s_{\alpha}^{L}, r_{\alpha}^{L} s_{\alpha}^{R}, r_{\alpha}^{R} s_{\alpha}^{L}, r_{\alpha}^{R} s_{\alpha}^{R}\right), \max \left(r_{\alpha}^{L} s_{\alpha}^{L}, r_{\alpha}^{L} s_{\alpha}^{R}, r_{\alpha}^{R} s_{\alpha}^{L}, r_{\alpha}^{R} s_{\alpha}^{R}\right)\right]
$$

4. Division:

$$
\begin{aligned}
(\widetilde{r}: \widetilde{s})_{\alpha}= & {\left[r_{\alpha}^{L}, r_{\alpha}^{R}\right]:\left[s_{\alpha}^{L}, s_{\alpha}^{R}\right] } \\
= & {\left[\min \left(\frac{r_{\alpha}^{L}}{s_{\alpha}^{L}}, \frac{r_{\alpha}^{L}}{s_{\alpha}^{R}}, \frac{r_{\alpha}^{R}}{s_{\alpha}^{L}}, \frac{r_{\alpha}^{R}}{s_{\alpha}^{R}}\right), \max \left(\frac{r_{\alpha}^{L}}{s_{\alpha}^{L}}, \frac{r_{\alpha}^{L}}{s_{\alpha}^{R}}, \frac{r_{\alpha}^{R}}{s_{\alpha}^{L}}, \frac{r_{\alpha}^{R}}{s_{\alpha}^{R}}\right)\right], } \\
& \text { except for } s_{\alpha}^{L} \leq 0 \leq s_{\alpha}^{R} .
\end{aligned}
$$

If $\widetilde{r}, \widetilde{s} \in F\left(\mathbb{R}_{+}\right)$, then $(\widetilde{r} \cdot \widetilde{s})_{\alpha}=\left[r_{\alpha}^{L} s_{\alpha}^{L}, r_{\alpha}^{R} s_{\alpha}^{R}\right], \forall \alpha \in[0.5,1]$. 
And if, $\widetilde{r} \in F\left(\mathbb{R}_{+}\right)$and $\widetilde{s} \in F\left(\mathbb{R}_{++}\right)$, then

$$
(\widetilde{r}: \widetilde{s})_{\alpha}=\left[\frac{r_{\alpha}^{L}}{s_{\alpha}^{R}}, \frac{r_{\alpha}^{R}}{s_{\alpha}^{L}}\right], \quad \forall \alpha \in[0.5,1] .
$$

Vector case, let $\tilde{r} \in F^{n}(\mathbb{R})=F(\mathbb{R}) \times F(\mathbb{R}) \times \cdots \times F(\mathbb{R})$, i.e. $\widetilde{r}=\left(\widetilde{r}_{1}, \cdots, \widetilde{r}_{n}\right)$, where $\tilde{r}_{i} \in F(\mathbb{R}), \forall i=1,2, \cdots, n$, we define $r_{\alpha}^{L}=\left(r_{1 \alpha}^{L}, \cdots, r_{n \alpha}^{L}\right)^{T}$ and $r_{\alpha}^{R}=$ $\left(r_{1 \alpha}^{R}, \cdots, r_{n \alpha}^{R}\right)^{T}$. The order relation between two fuzzy vectors $\widetilde{r}, \widetilde{s} \in F^{n}(\mathbb{R})$ is defined as follows: $\widetilde{r} \preceq_{\alpha} \widetilde{s}$ iff $\widetilde{r}_{i} \preceq_{\alpha} \widetilde{s}_{i} \forall i=1,2, \cdots, n$.

On account of Proposition 3.1, it follows that $\widetilde{r} \preceq_{\alpha} \widetilde{s}$ iff $\widetilde{r}_{i \alpha}^{L} \leq \widetilde{s}_{i \alpha}^{L}$ and $\widetilde{r}_{i \alpha}^{R} \leq \widetilde{s}_{i \alpha}^{R}$ $\forall i=1,2, \cdots, n \forall \alpha \in[0.5,1]$.

Suppose $\widetilde{r} \in F^{n}(\mathbb{R}), x \in \mathbb{R}^{n}, x \geq 0$. Then the product $(\widetilde{r} x)$ is a fuzzy number whose $\alpha$-cut is given by $[(\widetilde{r} x)]_{\alpha}=\left[(\widetilde{r} x)_{\alpha}^{L},(\widetilde{r} x)_{\alpha}^{R}\right]=\left[\left[\left(\widetilde{r}_{\alpha}^{L}\right)^{T} x,\left(\widetilde{r}_{\alpha}^{R}\right)^{T} x\right]\right.$.

Now consider the problem (FLP):

Let $S=\left\{x: x \in R^{n}, x=0\right.$ or $\left.1, \sum a_{i j} x_{j} \geq 1, i=1, \cdots, m, j=1, \cdots, n\right\}$ be the feasible set of (FLP).

Let $\alpha \in[0.5,1]$ be the grade of satisfaction associated with the fuzzy objective function of (FLP). For consistency with the ranking relation defined earlier we assume that $\alpha$ lie in the interval $[0.5,1]$. As for convention we assume that, $(\widetilde{c} x+$ $\widetilde{p}) \in F\left(\mathbb{R}_{+}\right)$and $(\widetilde{d} x+\widetilde{q}) \in F\left(\mathbb{R}_{++}\right), \forall x \in S, \forall \alpha \in[0.5,1]$.

Definition 3.2 ( $\alpha$-Optimal Solution). A vector $x_{\alpha}^{*} \in S$ is said to be an $\alpha$-optimal solution of the problem (FLP) if there does not exist any $x \in S$ such that $\widetilde{z}(x) \prec_{\alpha}$ $\widetilde{z}\left(x_{\alpha}^{*}\right)$. (Ref. [7])

Since $x \geq 0$, the problem (FLP) can be written as

$(\mathbf{F L P})_{\alpha} \operatorname{Min} f_{\alpha}(x)=\left(\sum_{j=1}^{n}\left[c_{j \alpha}^{L}, c_{j \alpha}^{R}\right] x_{j}+\left[p_{\alpha}^{L}, p_{\alpha}^{R}\right]\right):\left(\sum_{j=1}^{n}\left[d_{j \alpha}^{L}, d_{j \alpha}^{R}\right] x_{j}+\left[q_{\alpha}^{L}, q_{\alpha}^{R}\right]\right)$ subject to $x \in S$.

Taking $f_{\alpha}(x)=\left[f_{\alpha}^{L}(x), f_{\alpha}^{R}(x)\right]$, and using the division operation of fuzzy numbers, $(\mathbf{F L P})_{\alpha}$ reduces to an equivalent bi-objective programming problem $(\mathbf{B O P})_{\alpha}$ (Ref. [7])

$(\mathrm{BOP})_{\alpha}$

$$
\min \left(f_{\alpha}^{L}(x), f_{\alpha}^{R}(x)\right)=\left(\frac{\sum_{j=1}^{n} c_{j \alpha}^{L} x_{j}+p_{\alpha}^{L}}{\sum_{j=1}^{n} d_{j \alpha}^{R} x_{j}+q_{\alpha}^{R}}, \frac{\sum_{j=1}^{n} c_{j \alpha}^{R} x_{j}+p_{\alpha}^{R}}{\sum_{j=1}^{n} d_{j \alpha}^{L} x_{j}+q_{\alpha}^{L}}\right)
$$

subject to $x \in S$.

Remark 3.3. For $\alpha \in[0.5,1]$, a vector $x_{\alpha}^{*} \in S$ is an $\alpha$-optimal solution of the problem (FLP) if there does not exist any $x \in S$ such that $\left(f_{\alpha}^{L}\left(x_{\alpha}^{*}\right), f_{\alpha}^{R}\left(x_{\alpha}^{*}\right)\right)>$ $\left(f_{\alpha}^{L}(x), f_{\alpha}^{R}(x)\right)$, i.e. $x_{\alpha}^{*}$ is a weakly efficient solution (Ref. [8]) of the bi-objective set covering problem $(\mathbf{B O P})_{\alpha}$. 
We now associate two linear fractional set covering problems $(\mathbf{L F P})_{\alpha}$ and $(\mathbf{R F P})_{\alpha}$ with $(\mathbf{B O P})_{\alpha}$, for every $\alpha \in[0.5,1]$. (Ref. [7])

$(\mathbf{L F P})_{\alpha}$

$$
\operatorname{Min} f_{\alpha}^{L}(x)=\left(\frac{\sum_{j=1}^{n} c_{j \alpha}^{L} x_{j}+p_{\alpha}^{L}}{\sum_{j=1}^{n} d_{j \alpha}^{R} x_{j}+q_{\alpha}^{R}}\right)
$$

subject to $x \in S$

$(\mathbf{R F P})_{\alpha}$

$$
\operatorname{Min} f_{\alpha}^{R}(x)=\left(\frac{\sum_{j=1}^{n} c_{j \alpha}^{R} x_{j}+p_{\alpha}^{R}}{\sum_{j=1}^{n} d_{j \alpha}^{L} x_{j}+q_{\alpha}^{L}}\right)
$$

subject to $x \in S$

Let $x_{\alpha}^{L}$ and $x_{\alpha}^{R}$ be, respectively, the optimal solutions of $(\mathbf{L F P})_{\alpha}$ and $(\mathbf{R F P})_{\alpha}$. Then, for any $x \in S$, we have

$$
\left(f_{\alpha}^{L}(x), f_{\alpha}^{R}(x)\right) \geq\left(f_{\alpha}^{L}\left(x_{\alpha}^{L}\right), f_{\alpha}^{R}\left(x_{\alpha}^{R}\right)\right)
$$

and

$$
\begin{aligned}
& \left(f_{\alpha}^{L}\left(x_{\alpha}^{R}\right), f_{\alpha}^{R}\left(x_{\alpha}^{R}\right)\right) \ngtr\left(f_{\alpha}^{L}(x), f_{\alpha}^{R}(x)\right) \\
& \left(f_{\alpha}^{L}\left(x_{\alpha}^{L}\right), f_{\alpha}^{R}\left(x_{\alpha}^{L}\right)\right) \ngtr\left(f_{\alpha}^{L}(x), f_{\alpha}^{R}(x)\right) .
\end{aligned}
$$

Thus $x_{\alpha}^{L}$ and $x_{\alpha}^{R}$ are weakly efficient solutions of the bi-objective set covering problem $(\mathbf{B O P})_{\alpha}$. Moreover, since $x_{\alpha}^{L}, x_{\alpha}^{R} \in S$ so we also have

$$
f_{\alpha}^{L}\left(x_{\alpha}^{L}\right) \leq f_{\alpha}^{L}\left(x_{\alpha}^{R}\right) \leq f_{\alpha}^{R}\left(x_{\alpha}^{R}\right) \leq f_{\alpha}^{R}\left(x_{\alpha}^{L}\right)
$$

Remark 3.4. It may be noted that the $\alpha$-interval of confidence $\left[\left(f_{\alpha}^{L}\left(x_{\alpha}^{L}\right), f_{\alpha}^{R}\left(x_{\alpha}^{L}\right)\right]\right.$ of $(\mathbf{L F P})_{\alpha}$ evaluated at $x_{\alpha}^{L}$ is the superset of the $\alpha$-interval of confidence $\left[\left(f_{\alpha}^{L}\left(x_{\alpha}^{R}\right), f_{\alpha}^{R}\left(x_{\alpha}^{R}\right)\right]\right.$ of $(\mathbf{R F P})_{\alpha}$ evaluated at $x_{\alpha}^{R}$. From the decision maker view point, the larger $\alpha$-interval of confidence offers more flexibility leading to more acceptability.

The above remark along with Definition 3.1 motivated us to define the following new concept.

Definition 3.5. For $\alpha \in[0.5,1]$, the $x_{\alpha}^{L}$ vector is called an $\alpha$-acceptable optimal solution of (FLP). The corresponding objective function value, $\widetilde{f}\left(x_{\alpha}^{L}\right)=$ $\left[\left(f_{\alpha}^{L}\left(x_{\alpha}^{L}\right), f_{\alpha}^{R}\left(x_{\alpha}^{L}\right)\right]\right.$, is called an $\alpha$-acceptable optimal value of the problem (FLP).

It is important to point out here that the Definition 3.2 takes in to consideration the crisp scenario when $\alpha=1$. 
Now to find the optimal solution for

$(\mathbf{L F P})_{\alpha}$

$$
\operatorname{Min} f_{\alpha}^{L}(x)=\left(\frac{\sum_{j=1}^{n} c_{j \alpha}^{L} x_{j}+p_{\alpha}^{L}}{\sum_{j=1}^{n} d_{j \alpha}^{R} x_{j}+q_{\alpha}^{R}}\right)
$$

subject to $x \in S$

we need to show that the objective function of $(\mathbf{L F P})_{\alpha}$ to be pseudo-convex. This is proved in the following theorem.

Theorem 3.6. The objective function of $(\mathbf{L F P})_{\alpha}$ is pseudo-convex.

Proof. Since $\sum_{j=1}^{n} d_{j \alpha}^{R} x_{j}+q_{\alpha}^{R}$ and $\sum_{j=1}^{n} c_{j \alpha}^{L} x_{j}+p_{\alpha}^{L}$ are linear and, therefore, convex functions, we conclude that $\frac{\sum_{j=1}^{n} c_{j \alpha}^{L} x_{j}+p_{\alpha}^{L}}{\sum_{j=1}^{n} d_{j \alpha}^{R} x_{j}+q_{\alpha}^{R}}$ is a pseudo-convex function.

The following algorithm is constructed to enumerate the given problem:

\section{Algorithm.}

Step 1. Given (FLP) form the corresponding parametric programming problem $(\mathbf{R F P})_{\alpha}$ and $(\mathbf{L F P})_{\alpha}$ with parameter $\alpha$.

Step 2. Choose any value of the parameter $\alpha$ lying in the interval $[0.5,1]$ so that $(\mathbf{L F P})_{\alpha}$ converts to (LFP).

Step 3. Associated with (LFP) form the corresponding continuous program (LFP') by embedding the feasible region of (LFP) into $R^{n}$ (a cube with $n$ vertices). Let $S$ be the feasible set for (LFP').

Step 4. Choose a feasible solution $x_{0} \in S$ such that $\nabla f\left(x_{0}\right) \neq 0$. Form the corresponding linear program (LP). On solving (LP) let $x_{1}$ be its optimal solution. If $x_{1}=x_{0}$ and of the $0-1$ form then this is the required solution of the given problem, otherwise let $S_{1}=\left\{x_{1}\right\}$.

Step 5. Starting with the point $x_{1}$, form corresponding (LP), let its optimal solution be $x_{2} \neq x_{1}$. Update $S_{1}$ i.e. $S_{1}=\left\{x_{1}, x_{2}\right\}$. Step 6. Repeat Step 5 for the point $x_{2}$ and suppose at the $i$ th stage $S_{1}=\left\{x_{1}, x_{2}, \ldots, x_{i}\right\}$.

Stop if at the $(i+1)$ th stage $x_{i+1} \in S_{i}$, then $x_{i+1}$ is the optimal solution of (LFP').

Step 7. If $x_{i+1}$ is an optimal solution of the form $0-1$ then it is a solution of (LFP) otherwise, go to Step 8.

Step 8. Apply Gomory cuts to find a solution of the $0-1$ form and the corresponding prime cover.

Step 9. Suppose optimal solution of (LFP) be $x^{*}$ and $x_{l}^{*}, x_{r}^{*}$ be the corresponding optimal value of $(\mathbf{L F P})_{\alpha}$ and $(\mathbf{R F P})_{\alpha}$ corresponding to $x^{*}$ respectively, then the optimal solution for the given $(\mathbf{F L P})$ be $x^{*}$ with optimal value $\left[x_{l}^{*}, x_{r}^{*}\right]$. 
Note. The algorithm must terminate after finite number of steps as it moves only on the vertices of the feasible region, which are finite in numbers, i.e. convergence is must.

\section{NumERicAl EXAMPLE}

To illustrate the efficiency of the proposed method consider the following examples:

Example 1. Consider the problem (FLP)

$$
\begin{array}{rc}
\min f(x)= & \frac{\widetilde{c}_{1} x_{1}+\widetilde{c}_{2} x_{2}+\widetilde{c}_{3} x_{3}+\widetilde{p}}{\widetilde{d}_{1} x_{1}+\widetilde{d}_{2} x_{2}+\widetilde{d}_{3} x_{3}+\widetilde{q}} \\
\text { subject to } \quad & x_{1}+x_{2} \geq 1 \\
& x_{2}+x_{3} \geq 1 \\
& x_{1}+x_{3} \geq 1 \\
& x_{i}=0 \text { or } 1, \quad i=1,2,3
\end{array}
$$

where the membership functions of $\widetilde{c}_{1}, \widetilde{c}_{2}, \widetilde{c}_{3}, \widetilde{p}, \widetilde{d}_{1}, \widetilde{d}_{2}, \widetilde{d}_{3}$ and $\widetilde{q}$ are

$$
\begin{aligned}
& \widetilde{c}_{1}(x)=\left\{\begin{array}{ll}
0 & x<1 \\
x-5 & 5 \leq x \leq 6 \\
1 & 6 \leq x \leq 7 \\
(20-x) / 13 & 7<x \leq 20 \\
0 & 20<x
\end{array}, \quad \widetilde{c}_{2}(x)= \begin{cases}0 & x<16 \\
x-16 & 16 \leq x \leq 17 \\
1 & 17 \leq x \leq 18 \\
(40-x) / 22 & 18<x \leq 40 \\
0 & 40<x\end{cases} \right. \\
& \widetilde{c}_{3}(x)=\left\{\begin{array}{ll}
0 & x<24 \\
x-24 & 24 \leq x \leq 25 \\
1 & 25 \leq x \leq 26 \\
(50-x) / 24 & 26<x \leq 50 \\
0 & 50<x
\end{array} \quad \widetilde{p}(x)= \begin{cases}0 & x<2 \\
(x-2) / 3 & 2 \leq x<5 \\
(9-x) / 4 & 5 \leq x \leq 9 \\
0 & x \geq 9\end{cases} \right. \\
& \widetilde{d}_{1}(x)=\left\{\begin{array}{ll}
0 & x<4 \\
(x-4) / 2 & 4 \leq x<6 \\
(8-x) / 2 & 6 \leq x \leq 8 \\
0 & x \geq 8
\end{array}, \quad \widetilde{d}_{2}(x)= \begin{cases}0 & x<5 \\
(x-5) / 2 & 5 \leq x<7 \\
(12-x) / 5 & 7 \leq x \leq 12 \\
0 & x \geq 12\end{cases} \right. \\
& \widetilde{d}_{3}(x)=\left\{\begin{array}{ll}
0 & x<6 \\
(x-6) / 5 & 6 \leq x<11 \\
(13-x) / 2 & 11 \leq x \leq 13 \\
0 & x \geq 13
\end{array}, \quad \widetilde{q}(x)= \begin{cases}0 & x<15 \\
x-15 & 15 \leq x<16 \\
17-x & 16 \leq x \leq 17 \\
0 & x \geq 17\end{cases} \right.
\end{aligned}
$$


Step 1. To solve the above problem, the following $(\mathbf{L F P})_{\alpha}$ and $(\mathbf{R F P})_{\alpha}$ problems are considered

$$
\begin{aligned}
&(\mathbf{L F P})_{\alpha} \quad \min z(x)= \frac{(\alpha+5) x_{1}+(\alpha+16) x_{2}+(\alpha+24) x_{3}+3 \alpha+2}{(8-2 \alpha) x_{1}+(12-5 \alpha) x_{2}+(13-2 \alpha) x_{3}+17-\alpha} \\
& \text { subject to } x_{1}+x_{2} \geq 1 \\
& x_{2}+x_{3} \geq 1 \\
& x_{1}+x_{3} \geq 1 \\
& x_{i}=0 \text { or } 1 ; \quad i=1,2,3 . \\
&(\mathbf{R F P})_{\alpha} \quad \min z(x)= \frac{(20-13 \alpha) x_{1}+(40-22 \alpha) x_{2}+(50-24 \alpha) x_{3}-4 \alpha+9}{(2 \alpha+4) x_{1}+(2 \alpha+5) x_{2}+(5 \alpha+6) x_{3}+\alpha+15} \\
& \operatorname{subject~to~} x_{1}+x_{2} \geq 1 \\
& x_{2}+x_{3} \geq 1 \\
& x_{1}+x_{3} \geq 1 \\
& x_{i}=0 \text { or } 1 ; \quad i=1,2,3 .
\end{aligned}
$$

Step 2. Put $\alpha=1 / 2$, above problem reduces to

(LFP)

$$
\begin{aligned}
\min z(x)= & \frac{11 x_{1}+33 x_{2}+49 x_{3}+7}{14 x_{1}+19 x_{2}+24 x_{3}+33} \\
\text { subject to } & x_{1}+x_{2} \geq 1 \\
& x_{2}+x_{3} \geq 1 \\
& x_{1}+x_{3} \geq 1 \\
& x_{i}=0 \text { or } 1 ; \quad i=1,2,3 .
\end{aligned}
$$

where $J=\{1,2,3\}, I=\{1,2,3\}$.

Step 3. The corresponding (LFP') is

$$
\begin{aligned}
\operatorname{Min} f(x) & =\frac{11 x_{1}+33 x_{2}+49 x_{3}+7}{14 x_{1}+19 x_{2}+24 x_{3}+33} \\
X & =\left(x_{1}, x_{2}, x_{3}\right) \in S=\left\{\left(x_{1}, x_{2}, x_{3}\right) \mid x_{1}+x_{2}\right. \\
& \left.\geq 1, x_{1}+x_{3} \geq 1, x_{1}, x_{2}, x_{3} \geq 0\right\}
\end{aligned}
$$

Step 4. Choose $X_{0}=(1,1,0)$ as one of the feasible solution of (LFP') with $\nabla f\left(X_{0}\right) \neq 0$.

The corresponding (LP) is

$$
\text { Minimize } \nabla f\left(X_{0}\right)^{T} X=0.003 x_{1}+0.278 x_{2}+0.416 x_{3}: X \in S \text {. }
$$


After applying the simplex algorithm the final optimal table is as follows:

\begin{tabular}{|c|c|c|c|c|c|c|c|c|}
\hline & & $C_{j}$ & -0.003 & -0.278 & -0.461 & 0 & 0 & 0 \\
\hline$C_{B}$ & $B$ & $X_{B}$ & $Y_{1}$ & $Y_{2}$ & $Y_{3}$ & $S_{1}$ & $S_{2}$ & $S_{3}$ \\
\hline-0.003 & $X_{1}$ & 1 & 1 & 0 & 1 & 0 & 0 & -1 \\
\hline-0.278 & $X_{2}$ & 1 & 0 & 1 & 1 & 0 & -1 & 0 \\
\hline 0 & $S_{1}$ & 1 & 0 & 0 & 2 & 1 & -1 & -1 \\
\hline & & -0.281 & 0 & 0 & 0.18 & 0 & 0.278 & 0.003 \\
\hline
\end{tabular}

Hence the optimal solution of $(\mathbf{L P})$ is $x_{1}=(1,1,0)$, which is equal to $x_{0}=(1,1,0)$ also of the $0-1$ form, therefore this is the optimal solution for the (LFP) with optimal value 0.77 .

Step 5. The optimal values of $(\mathbf{L F P})_{\alpha}$ and $(\mathbf{R F P})_{\alpha}$ corresponding to $x_{1}=$ $(1,1,0)$ are 0.77 and 1.87 respectively. Hence the optimal value for the given (FLP) is $f(x)=[0.77,1.87]$.

\section{Conclusion}

In this paper a solution technique to solve a linear fractional set covering problem with fuzzy parameters in the objective function is being given. Given a fuzzy linear fractional set covering problem, the corresponding bi-objective set covering problem is formed. Then to solve this problem a linearization technique is being given. Obviously this is not the only way to solve the problem considered and therefore one can assure that according to both the interests of the decision maker and the nature of the problem. Different methods of solution may be used to obtain optimal solutions. More importantly, this approach gives the fuzzy solution to the fuzzy problem, which is very useful for the decision maker since it provides the range in the solution.

Acknowledgements. The authors are grateful to the referees for their valuable comments and suggestions.

\section{REFERENCES}

[1] D. Dubois and H. Prade, Ranking fuzzy numbers in the setting of possibility theory. Inf. Sci. 30 (1983) 183-224.

[2] M.J. Hwang, C.I. Chiang and Y.H. Liu, Solving a fuzzy set covering problem. Math. Comput. Model. 40 (2004) 861-865.

[3] A. Kaufman and M.M. Gupta, Fuzzy Mathematical Models in Engineering and Management Sciences. Elsevier Science Publishers B.V., Amsterdam (1988). 
[4] A. Kaufman and M.M. Gupta, Introduction to fuzzy arithmetic, theory and applications. New York, Von Nostrand Reinhold (1991).

[5] J. Li and Raymond S.K. Kwan, A meta-heuristic with orthogonal experiment for the set covering problem. J. Math. Model. Algorithms 3 (2004) 263-283.

[6] J. Li and Raymond S.K. Kwan, A fuzzy evolutionary approach with Taguchi parameter setting for the set covering problem. School of Computing, University of Leads LS 2 9JT, UK, 2002.

[7] A. Mehra, S. Chandra and C.R. Bector, Acceptable optimality in linear fractional programming with fuzzy coefficients. Fuzzy Optim. Decis. Making 6 (2007) 6-16.

[8] B. Metev and D. Gueorguieva, A simple method for obtaining weakly efficient points in multiobjective linear fractional programming problems. Eur. J. Oper. Res. 12 (2000) 325338 .

[9] R. Sahraeian and M.S. Kazemi, A fuzzy set covering-clustering algorithm for facility location problem. IEEE International Conference on Industrial Engeneering Management (2011) 1098-1102.

[10] R.R. Saxena and S.R. Arora, A linearization technique for solving the quadratic set covering problem. Optimization 39 (1996) 35-42.

[11] R.R. Saxena and R. Gupta, Enumeration technique for solving linear fractional fuzzy set covering problem, Int. J. Pure Appl. Math. 84 (2013) 477-496.

[12] R.R. Saxena and R. Gupta, Enumeration technique for solving linear fuzzy set covering problem, Int. J. Pure Appl. Math. 85 (2013) 635-651.

[13] H. Shavandi and H. Mahlooji, Fuzzy hierarchical queueing models for the location set covering problem in congested systems. Scientia Iranica 15 (2008) 378-388.

[14] B. Stanojević and M. Stanojević, Solving method for linear fractional optimization problem with fuzzy coefficients in the objective fucntion. Int. J. Comput. Commun. 8 (2013) 146-152.

[15] H.C. Wu, Duality theorems in fuzzy mathematical programming problems based on the concept of necessity. Fuzzy Sets and Systems 139 (2003) 363-377.

[16] H.J. Zimmermann, Fuzzy set theory and its applications. 4th edition, Nowell, MA Kluwer Academic Publishers (2001).

[17] K. Zimmermann, Fuzzy set covering problem. Int. J. General Syst. 20 (1991) 127-131. 